\title{
Distributed Robust Model Predictive Control- Based Energy Management Strategy for Islanded Multi-Microgrids Considering Uncertainty
}

\author{
Zhuoli Zhao, Member, IEEE, Juntao Guo, Xi Luo, Chun Sing Lai, Senior Member, IEEE, Ping Yang, \\ Loi Lei Lai, Fellow, IEEE, Peng Li, Josep M. Guerrero, Fellow, IEEE, and Mohammad Shahidehpour, \\ Life Fellow, IEEE
}

\begin{abstract}
A microgrid is considered to be a smart power system that can integrate local renewable energy effectively. However, the intermittent nature of renewable energy causes operating pressure and additional expense in maintaining the stable operation by the energy management system in a microgrid. The structure of multimicrogrids provides the possibility to construct flexible and various energy trading framework. In this paper, in order to reduce the adverse effects of uncertain renewable energy output, a distributed robust model predictive control (DRMPC)-based energy management strategy is proposed for islanded multi-microgrids. This strategy balances the robustness and economy of singlemicrogrid system operation by combining the advantages of robust optimization and model predictive control, while coping with the uncertainty of renewable energy sources. Furthermore, a dynamic energy trading market is formed among microgrids, which can enhance the overall economy of the multi-microgrids system. Simulation results verify the feasibility of the proposed DRMPC strategy.
\end{abstract}

Index Terms-Multi-microgrids, energy management, distributed robust model predictive control, game theory, renewable energy

\section{INTRODUCTION}

$\mathrm{T}^{\mathrm{h}}$ he renewable energy sources such as photovoltaic (PV) and wind turbine (WT) generation are connected to microgrids (MGs) as distributed generation (DG) units through the conversion of a series of power-electronic equipment to provide power for loads [1]. Compared with the traditional distribution network with centralized power generation, the needs of the rapid expansion of the power grid will be met by the closer electrical distance to the user side and the smaller line loss with these units. Moreover, the smaller environmental cost also helps the goal to lower carbon of energy transformation in power grid.

This work was supported by the National Natural Science Foundation of China 51907031; Guangdong Basic and Applied Basic Research Foundation (Guangdong-Guangxi Joint Foundation) 2021A1515410009; VILLUM FONDEN under the VILLUM Investigator Grant (no. 25920): Center for Research on Microgrids (CROM). (Corresponding authors: Chun Sing Lai; Loi Lei Lai)

Z. Zhao, J. Guo, X. Luo, and L. L. Lai are with the Department of Electrical Engineering, School of Automation, Guangdong University of Technology, Guangzhou, 510006, China (e-mail: zhuoli.zhao@gdut.edu.cn; 2111904003@mail2.gdut.edu.cn; 2112004004@mail2.gdut.edu.cn; 1.1.lai@ieee.org).

C. S. Lai is with Brunel Interdisciplinary Power Systems (BIPS) Research Centre, Electronic and Electrical Engineering, Brunel University London, London, UB8 3PH, UK and also with the Department of Electrical Engineering,
The microgrid is regarded as a platform for regional management of these flexible distributed devices to ensure the balance of power supply and demand. Nevertheless, in the face of the MG with high penetration rates of renewable energy, it is still a big problem and worth discussing to ensure the effective operation of energy management strategy because of the volatility and uncertainty of natural sources.

In order to minimize the operation cost, the most economical operation decisions can be made with traditional day-ahead scheduling strategy. However, the accuracy of the strategy is constrained by the precision of the forecast output of renewable energy. Operators of MGs often inevitably need to pay more for the inaccurate predictions to ensure the stable operation of the MGs. Therefore, the strategies are designed more inclined to prevent the uncertainty of renewable energy in advance, which are more suitable for the flexible characteristics of MGs.

The worst scenario is taken into account by the robust optimization (RO) strategy [2]. Therefore, RO can ensure that there is still a feasible solution even when the uncertain variable fluctuates within a certain range [3]. But this conservative solution will inevitably sacrifice a part of the economy [4]-[7]. In [4], a scenario-based robust energy management method was developed which the possible testing scenarios are provided by Taguchi's orthogonal array testing method. In [5], a hybrid stochastic/robust optimization model was proposed to minimize the expected net cost considering the uncertainty of real-time market price. The research gap that cogeneration equipment cannot be effectively coordinated with industrial demand response on different time scales is filled with an adaptive robust approach in [6]. In [7], a short-term scheduling problem of integrated heat and power MG in the grid-connected mode was solved using RO method with uncertainty set. These studies

School of Automation, Guangdong University of Technology, Guangzhou, 510006, China (e-mail: chunsing.lai@brunel.ac.uk).

P. Yang is with the Guangdong Key Laboratory of Clean Energy Technology, South China University of Technology, Guangzhou 510640, China (e-mail: eppyang@scut.edu.cn).

P. Li is with the Digital Grid Research Institute of China Southern Power Grid, Guangzhou 510663, China (e-mail: lipeng@csg.cn).

J. M. Guerrero is with the Villum Center for Research on Microgrids (CROM), AAU Energy, Aalborg University, 9220 Aalborg East, Denmark (e-mail: joz@energy.aau.dk).

Mohammad Shahidehpour is with the Electrical and Computer Engineering Department, Illinois Institute of Technology, Chicago, IL, 60616, USA. He is also a Research Professor in the ECE Department at the King Abdulaziz University in Saudi Arabia (e-mail: ms@iit.edu). 
effectively warn MGs about forecast errors in advance by using different methods, which will increase the safety margin of the dispatch plan and the robustness of the energy management system (EMS) of MGs. However, some shortcomings of the RO strategy are exposed, such as larger computational burden and more insufficient flexibility.

The application of model predictive control (MPC) in EMS is based on the idea of closed-loop rolling optimization to predict and respond to the fluctuation of renewable energy in advance [8]-[13]. A multi-time scale optimal scheduling based on MPC in a designed three-tier coordinated scheduling system is shown in [9]. In [10], a hierarchical stochastic control scheme based on MPC was proposed for the coordination of plug-in electric vehicles (PEVs) charging and wind power. A closed-loop distributed model predictive control (DMPC) was designed in [11] to address the intra-hour economic dispatch problem, which can reduce potential variations in the determined generation schedules. In [12], the results of multiple uncertainty sampling were used to simulate the future characteristics of the converged $\mathrm{EV}$, and a two-layer control framework is proposed to optimize it. As a real-time strategy with multi-time scale, the flexibility of MPC provides a framework for combining with multiple methods of optimization. For example, a two-layer optimization model was proposed in [13] based on the robust model predictive control (RMPC) to deal with the uncertainty of renewable energy outputs in the distribution system restoration problem. The RMPC strategy can be regarded as a novel control strategy that combines the advantages of these two optimization methods, which can better weigh the robustness and economy in microgrids.

Furthermore, with the expansion of the number of MGs, operators tend to increase communication and interaction with nearby MGs to reduce more own operating costs. The emergence of multi-microgrids has laid a structural foundation for the cooperative operation of MGs, and also provides the possibility for the formation of free power market transactions among them [14]-[20]. An RO framework for joint an optimal scheduling of energy and reserves for multi-microgrids was proposed in [14], in which the non-anticipativity in reserve scheduling is considered. A decentralized robust model for optimal operation of the distribution company with private MGs was proposed in [15], in which the enforced operational uncertainties are handled by adaptive RO and the decentralized algorithm is solved by alternating direction method of multipliers (ADMM). In [16], two kinds of decentralized robust economic scheduling framework were reported based on analytical target cascading (ATC) method, which represents better information privacy and faster convergence rate respectively. The interconnected energy hubs $(\mathrm{EH})$ concept in networked microgrids was studied in [17], which aims to exploit the potential capabilities of microgrids in satisfying various types of energy demands. However, compared with intraday scheduling, the day-ahead trading strategy based on robust optimization cannot make timely adjustments to the uncertainty and trading mentality based on real-time weather changes and the enthusiasm of dealing with transactions for microgrid operators.

Similarly, based on the close and rapid information exchanges in the microgrid cluster, the real-time control strategies based MPC can be well applied in multi-microgrids energy management [18]-[20]. In [18], an online DMPC-based charging strategy for multiple EV charging stations was proposed in distribution systems, in which the online optimal charging problem is regarded as an optimal power flow problem that minimizes the total system energy cost. An optimal DMPC strategy to coordinate energy management among microgrid systems was presented in [19]; the renewable energy utilization is improved while maintaining its own systemwide supply and demand balance. In [20], an optimal procedure for the economic schedule was carried out through a control algorithm based on DMPC for networked interconnected microgrids with hybrid energy storage system. However, the complete trust in the forecast output of renewable energy will make the microgrids tend to make aggressive scheduling schemes in pursuit of economic which increase the high precision requirements of the forecasting method. Moreover, the lack of competition among microgrids in terms of transaction volume of energy and price makes these cooperative frameworks unattractive in the actual microgrid cluster.

For various microgrids with different ownerships, operators more often want to maximize their own profits through deep cooperation. Therefore, the multi-microgrids tend to find a transaction agreement that balances fairness and privacy [21][28]. In [21], a bi-level power management methodology based on approximate Reinforcement Learning was proposed to respond to the problem about the limited information cooperative agent can have from the MG asset behind the Point of Common Coupling (PCC). And a supervised multi-agent safe policy learning method for optimal power management was presented in [22] for networked microgrids considering $\mathrm{AC}$ power flow limits to ensure the safety of the control policy. In [23], a secure distributed transaction energy management scheme was proposed based on the collaborative optimization among microgrids with a misbehavior detection mechanism. A contribution-based energy trading mechanism among microgrids in a competitive market was designed in [24], in which the surplus energy will be allocated to consumers based on their historical contribution. However, the benefits and behaviors of seller who is the important participant in the power market have not been paid attention to. Based on [24], a method for fair and stable energy sharing among microgrid clusters with minimum information overhead was proposed in [25]. In this method, buyers will compete for the allocation with each other using priority factor based on a non-cooperative game. However, when the microgrid participates in the power market competition, its own energy management behavior is ignored, which cannot guarantee the maximization of the benefits of its operation and increases the risk of the safe operation of the microgrid. In [26], an agent-based hierarchical power management model was proposed, which is divided into a Nash bargaining game among MGs and an iterative machine learning mechanism on energy price for utility company. However, the wholesale price is the necessary reference that cannot be obtained in islanded multimicrogrids. In [27], a distributed collaborative energy bargain scheduling algorithm was designed based on a stochastic renewable power forecasting method. The trading behaviors are analyzed with the impacts of cooperation and dishonest behavior on the bargaining outcome. And as discussed in [25], this 


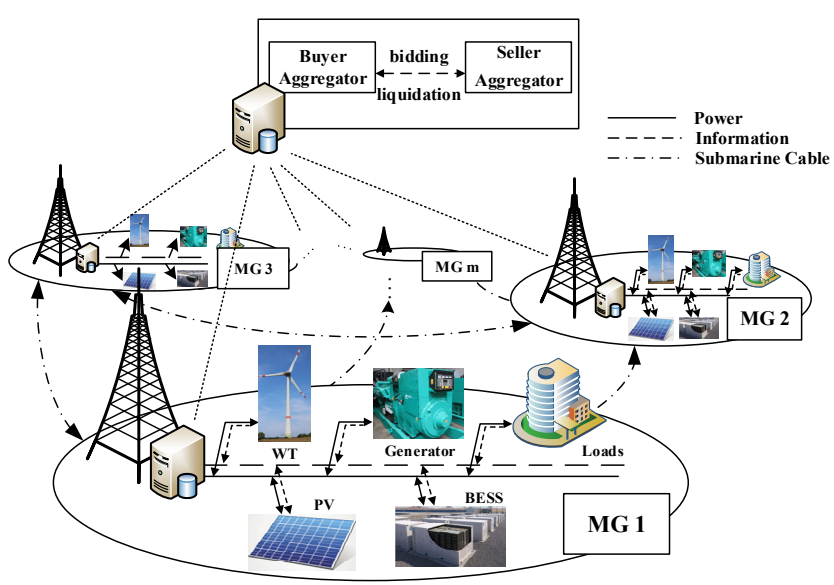

Fig. 1. The studied islanded multi-microgrids system.

cooperative method of cost allocation is affected by the selfish behavior of users driven by the interests, which is inevitable. In [28], the problem of bargaining was decomposed into two sequential problems as minimizing social cost and sharing of transaction benefits, which an incentive mechanism is designed to encourage active energy trading and fair benefit sharing based on dissatisfaction cost.

Most of the above research work focuses on the optimization of the overall cooperative economy, which requires a high degree of trust among microgrids to ensure the fairness of the strategy. And the main grid is one of the important participants in the strategy, who is difficult to connect for multi-microgrids operating in islanded scenario (i.e., remote island groups, rural areas, mountainous areas, and grassland.) In addition, under most competitive frameworks, transactions are only driven by price to pursue revenue, which there should be more behavioral choices for operators. Therefore, for more complex and changeable business relationships in the actual islanded multimicrogrids, it is more suitable to establish a more flexible framework with interactions of cooperative and non-cooperative game where the multiple businesses coexist.

In summary, the main contributions of this paper are as follows:

1) In response to the uncertainty of renewable energy, the microgrid is modeled based on RMPC with closed-loop feedback mechanism. Operators have more options in real-time to choose the operation plan considering the balance of robustness and economy.

2) By accounting for the complex business relationship with multiple owners in islanded multi-microgrids, a dynamic alliance transaction framework for multi-microgrids system is established. And a transaction game mechanism based on individual contribution is proposed to enhance the enthusiasm of members.

3) Combined with the ATC method, a distributed robust model predictive control framework is realized by the necessary information exchanging which respect privacy. On the basis of fair trading under the framework, the economy of the entire multi-microgrids system is enhanced.

\section{Robust Model Predictive Control Strategy For SINGLE MICROGRID}

\section{A. Dynamic Model for Single Microgrid}

The research in this paper is based on the islanded operation of multi-microgrids, so a multi-microgrids system located on a remote island group shown in Fig. 1 is regarded as an example in this paper. Each microgrid is equipped with its own power generation equipment on its own island, and needs to supply a certain number of power users for normal life or production. Considering the geographical location of island, the microgrids are not connected to the main grid on land. And the power transmission channels are provided by submarine cables among these microgrids.

\section{1) Renewable energy generation units}

The island microgrid is considered to be a typical renewable energy microgrid with high penetration. Especially for PV and WT, the actual output of power is greatly affected by natural resources that change in real time. Therefore, the uncertainty set of them is described as follows:

$$
\left\{\begin{array}{l}
P_{i}^{v}(k)=P_{i}^{v f}(k)+\alpha_{i, k}^{v} \cdot\left(\beta_{i, k}^{v u}-\beta_{i, k}^{v d}\right) \cdot P_{i}^{v e}(k) \\
P_{i}^{w}(k)=P_{i}^{w f}(k)+\alpha_{i, k}^{w} \cdot\left(\beta_{i, k}^{w u}-\beta_{i, k}^{w d}\right) \cdot P_{i}^{w e}(k)
\end{array}\right.
$$

where $k$ is the sampling time; $P_{i}^{v}$ and $P_{i}^{w}$ are the robust predictive output of PV and WT for MG $i ; P_{i}^{v f}, P_{i}^{w f}$ are the day-ahead predictive output; $P_{i}^{v e}, P_{i}^{\text {we }}$ are the allowable range of predictive error; $\alpha_{i}^{v}$ and $\alpha_{i}^{w}$ are the adjustment factors; $\beta_{i}^{v u}, \beta_{i}^{v d}, \beta_{i}^{w u}, \beta_{i}^{w d} \in\{0,1\}$, which are the state factors for positive and negative error of PV and WT. And the constraints of these factors are shown as follows:

$$
\begin{gathered}
\left\{\begin{array}{l}
0 \leq \alpha_{i, k}^{v} \leq 1 \\
0 \leq \alpha_{i, k}^{w} \leq 1
\end{array}\right. \\
\left\{\begin{array}{l}
\sum_{k=1}^{M}\left(\beta_{i, k}^{v u}+\beta_{i, k}^{v d}\right) \leq \Gamma_{i}^{v} \\
\sum_{k=1}^{M}\left(\beta_{i, k}^{w u}+\beta_{i, k}^{w d}\right) \leq \Gamma_{i}^{w}
\end{array}\right. \\
\left\{\begin{array}{l}
\beta_{i, k}^{v u}+\beta_{i, k}^{v d} \leq 1 \\
\beta_{i, k}^{w u}+\beta_{i, k}^{w d} \leq 1
\end{array}\right.
\end{gathered}
$$

where $M$ represents the prediction layer. And $\Gamma_{i}^{v}, \Gamma_{i}^{w}$ are the overall uncertainty factor of prediction during period $M . P_{i}^{v f}$, $P_{i}^{w f}$ are taken as the equation constraints of the strategy without considering uncertainty, thus $P_{r o}=\Gamma / M$ indicates the probability of constraint violation [3].

2) Micro gas turbine generator set

In order to deal with the uncertainty of renewable energy, controllable micro-sources like gas turbines are needed to be equipped to balance the basic balance of supply and demand. Therefore, the dynamic mathematical model of the power generation units is described as follows:

$$
\begin{gathered}
P_{i}^{g}(k+1)=P_{i}^{g}(k)+\Delta P_{i}^{g}(k) \\
P_{i}^{g \min } \leq P_{i}^{g}(k+1) \leq P_{i}^{g \max }
\end{gathered}
$$




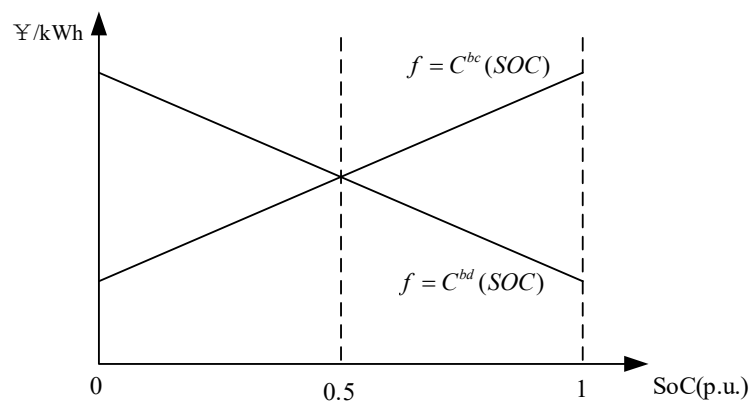

Fig. 2. The cost factor of BESS (SoC: State of Charge).

where $P_{i}^{g}$ is the supply amount of generator from MGi. $P_{i}^{g \min }$, $P_{i}^{g \max }$ are the lower and upper limit of $P_{i}^{g} . \Delta P_{i}^{g}$ indicates the adjustment value at time $k$, which is constrained by the ramping rate of the generator as

$$
\begin{gathered}
\Delta P_{i}^{g}(k)=\Delta P_{i}^{g u}(k)-\Delta P_{i}^{g d}(k) \\
\left\{\begin{array}{c}
0 \leq \Delta P_{i}^{g u}(k) \leq X_{i, k} \cdot \Delta P_{i}^{g \max } \\
0 \leq \Delta P_{i}^{g d}(k) \leq Y_{i, k} \cdot \Delta P_{i}^{g \max }
\end{array}\right. \\
X_{i, k}+Y_{i, k} \leq 1
\end{gathered}
$$

where $\Delta P_{i}^{g u}, \Delta P_{i}^{g d}$ are the adjustment value of up and down. $\Delta P_{i}^{g \text { max }}$ is the upper limit of adjustment. And $X_{i, k}, Y_{i, k} \in\{0,1\}$ are state variables.

3) Battery energy storage system

In order to consume renewable energy in time, the battery energy storage system (BESS) is regarded as a good choice to be auxiliary power supply equipment, which can balance economy and stability:

$$
S O C_{i}(k+1)=S O C_{i}(k)+\left(\eta_{i}^{c h} P_{i}^{b c}(k)-\frac{P_{i}^{b d}(k)}{\eta_{i}^{d i s}}\right) \cdot \Delta T
$$

where $S O C_{i}$ indicates the remaining energy of BESS. $P_{i}^{b c}, P_{i}^{b d}$ are the output of charge and discharge of battery. $\eta_{i}^{c h}, \eta_{i}^{d i s}$ are the efficiency factor. And $\Delta T$ is the running time of BESS. And the constraints of BESS are shown as follows.

$$
\left.\begin{array}{c}
S O C_{i}^{\min } \leq S O C(k+1) \leq S O C_{i}^{\max } \\
\left\{\begin{array}{l}
0 \leq P_{i}^{b c}(k) \leq W_{i, k} \cdot P_{i}^{b \max } \\
0 \leq P_{i}^{b d}(k) \leq Z_{i, k} \cdot P_{i}^{b \max }
\end{array}\right. \\
W_{i, k}+Z_{i, k} \leq 1
\end{array}\right\}
$$

where $S O C_{i}^{\min }, S O C_{i}^{\max }$ are the energy security limit for storage. $P_{i}^{b \max }$ is the upper limit of output of battery that limited by power electronics. $\mathrm{W}_{i, k}, \mathrm{Z}_{i, k} \in\{0,1\}$ are state variables. And Equation (14) indicates the constraint for balance of supply and demand in MG $i$.

\section{B. RMPC Prediction and Rolling Mechanism}

As we mentioned in Section I, the MPC strategy contains the idea of rolling optimization, which can be more flexibly

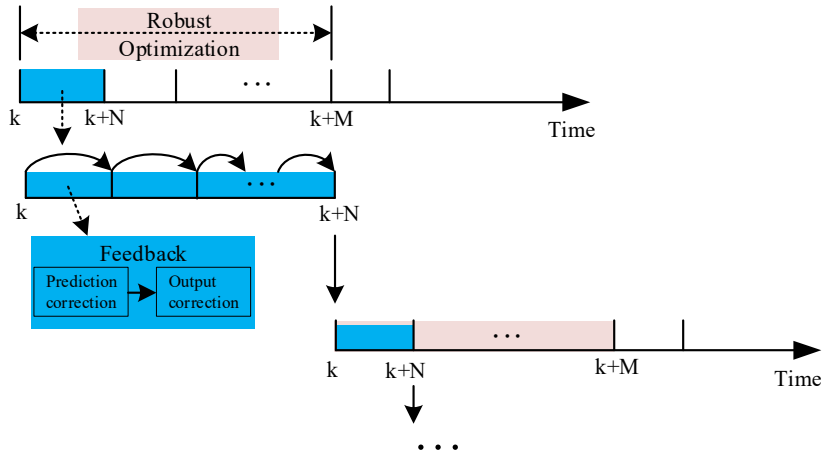

Fig. 3. The framework of RMPC.

integrated with other control strategies. Therefore, based on the renewable energy generation situation of the predicted layer length, the economic objective problem is transformed into a two-layer robust optimization problem to balance the robustness and economy of microgrid operation.

$$
\Phi_{m g}=\sum_{k=1}^{M} \min _{x}\left\{\max _{U} \min _{\Omega} f_{i}^{T} \cdot y_{i}\right\}
$$

Subject to: (16)-(17)

$$
\begin{gathered}
\left\{\begin{array}{l}
x=\{X, Y, Z, W\} \\
U=\left\{P^{v}, P^{w}\right\} \\
\Omega=\{x, U\}
\end{array}\right. \\
\left\{\begin{array}{l}
f_{i}=\left[\mathrm{C}_{i}^{b c}, \mathrm{C}_{i}^{b d}, \mathrm{C}_{i}^{g}, 0,0\right]^{\mathrm{T}} \\
y_{i}=\left[P_{i}^{b c}, P_{i}^{b d}, P_{i}^{g}, S O C_{i}, \Delta P_{i}^{g}\right]^{\mathrm{T}}
\end{array}\right.
\end{gathered}
$$

where $f_{i}$ is the set of cost coefficient. And $y_{i}$ indicates the set of scheduling plan.

It is worth mentioning that, in order to mobilize the enthusiasm of all power generation equipment in MG and also prevent excessive or low power reserves from damaging the battery, we designed a cost coefficient curve of the charging and discharging based on the remaining energy in a particular SOC.

$$
\left\{\begin{array}{l}
C_{i}^{b c}=A_{i} \cdot\left(S_{S O C_{i}}(k) / S O C_{i}^{c a p}\right)+C_{i} \\
C_{i}^{b d}=-A_{i} \cdot\left(S O C_{i}(k) / S O C_{i}^{c a p}\right)+B_{i}
\end{array}\right.
$$

where $\mathrm{SOC}_{i}^{\text {cap }}$ is the total capacity of BESS. $A_{i}, B_{i}$ and $C_{i}$ are the linear coefficient. The cost coefficient is fixed in the optimization within the prediction layer until the next rollover. And the relationship between the cost coefficient and the state of charge of BESS is shown in Fig. 2.

Finally, the control amount of length $N$ is inputted to the system until the next sampling time $k+N$.

\section{RMPC Feedback Mechanism}

Although there is an early warning for the forecast error of renewable energy in the robust optimization stage, the fluctuation of renewable energy is also very large in the ultrashort-term time frame. Therefore, the feedback mechanism is used to correct the deviation of smaller time scales that can form the closed-loop control in MPC. And the feedback mechanism is divided into prediction correction and output correction.

For the prediction correction, because there will be a certain error between the actual output and the predicted value, feedback of the actual output obtained in the past to the prediction model will correct the future predicted output. When the latest actual 
output is obtained, the prediction module in the feedback module will update the forecast change of renewable energy output at the next feedback point. That means the prediction module is continuously updated and rolled forward throughout the day.

This kind of prediction model generally adopts gray model or neural network, but the gray forecasting model is more sensitive to more regular simulation random fluctuation errors. Therefore, in order to cope with more complex real conditions, compared to our past work [29], this paper selects a convolutional neural network forecast model based on wavelet decomposition which is widely used in forecasting research.

And these prediction values will be inputted into the output correction. In the ultra-short period of time, if there is no advance redistribution strategy, the deviation between the forecast and the actual output will be borne by the generator, which poses a danger to the safe operation of the microgrid. Therefore, in the output correction, compared to pursue economy, the principle of redistribution is described as the minimal pressure on remaining power capacity.

$$
\left\{\begin{array}{l}
\operatorname{Min} \Delta P_{i}=\frac{\left|\Delta P_{i}^{g^{\prime}}\right|}{\left(P_{i}^{g \max }-P_{i}^{g}(k)\right)}+\frac{\left|\Delta P_{i}^{b^{\prime}}\right|}{\left(P_{i}^{b \max }-\left|P_{i}^{b}(k)\right|\right)} \\
\text { s.t.(5) - (14) }
\end{array}\right.
$$

where $\Delta P_{i}$ is the predicted deviation of the updated predicted value from the previous predicted point. And $\Delta P_{i}^{g \prime}, \Delta P_{i}^{b \prime}$ are the values redistributed to the power generation units and BESS, respectively.

Finally, the revised scheduling plan will be executed when the next feedback point arrives, and the feedback module will be executed in a loop until the next rolling layer sampling point arrives. The entire RMPC strategy framework based on time flow is shown in Fig. 3.

\section{RO solution mechanism}

As shown in Equation (3), $P_{R O}$ can be changed by adjusting the size of $\Gamma$, which be determined by the operators of the microgrid [3]. Therefore, the size of $\Gamma$ represents the degree of robustness and this kind of RO is called constraint robustness problem [30].

C\&CG algorithm is used to solve the RO problem. According to Equations (1)-(14) and (17), the dynamic model and the operation constraints of the microgrid can be transformed into the following standard form:

$$
\begin{gathered}
\left\{\begin{array}{l}
E_{i} y_{i} \leq j_{i}-F_{i} x_{i} \\
G_{i} y_{i} \leq g_{i} \\
H_{i} y_{i}=h_{i}
\end{array}\right. \\
\left\{\begin{array}{l}
I_{i} y_{i}=u_{i}^{v}, \quad u_{i}^{v} \in U \\
J_{i} y_{i}=u_{i}^{w}, \quad u_{i}^{w} \in U
\end{array}\right.
\end{gathered}
$$

Therefore, by decomposing the 'max-min-min' problem of Equation (15), the optimization problem can be divided into two stages, which the form of the main problem obtained is

$$
\begin{cases} & \min _{x} \Phi_{L B} \\ \text { s.t. } & \Phi_{L B} \geq \sum_{k=1}^{M} f_{i}^{T} \cdot y_{i} \\ & E q .(20) \\ & I_{i} y_{i}=u_{i}^{v^{*}}, \quad u_{i}^{v} \in U \\ & J_{i} y_{i}=u_{i}^{w^{*}}, \quad u_{i}^{w} \in U\end{cases}
$$

where $u_{i}^{v^{*}}$ and $u_{i}^{w^{*}}$ indicate the value of the uncertainty set obtained by the sub-problem after the $l$ th iteration.

And the form of the sub-problem is shown as

$$
\Phi_{U B}=\max _{U} \min _{\Omega} \sum_{k=1}^{M} f_{i}^{T} \cdot y_{i}
$$

The strong duality theory is used to linearize constraints, which makes the 'max-min' problem in (23) turn to a 'max' problem. The transformed dual problem with Lagrange duality variables is shown as follows:

$$
\left\{\begin{array}{l}
\Phi_{U B}=\max _{U}\left\{-\lambda^{T}\left(j_{i}-F_{i} x_{i}\right)-\mu^{T} g_{i}+\sigma^{T} h_{i}+\gamma^{T} u^{v}+\delta^{T} u^{w}\right\} \\
\left\{f_{i}^{T}+\lambda^{T} E_{i}+\mu^{T} G_{i}-\sigma^{T} H_{i}-\gamma^{T} I_{i}-\delta^{T} J_{i}\right\}=0 \\
\lambda \geq 0, \quad \mu \geq 0
\end{array}\right.
$$

At the beginning of the solution, the initial value $L B=-\infty$, $U B=+\infty$ and the number of iterations $l=1$ was set. Then the main-problem will be solved to send $x$ to sub-problem while $L B=\max \left(\mathrm{LB}_{l-1}, \Phi_{L B}\right)$ being updated. And the sub-problem will be solved to find the worst scenario $U$ for updated operating variables $x$ and update $U B=\min \left(\mathrm{UB}_{l-1}, \Phi_{U B}\right)$.

The new uncertain set $U$ with new constraints (20) created by variables $y_{i}^{l+1}$ will be sent back to the main problem to loop iteration until $U B-L B \leq \varepsilon^{R O}$ is satisfied, where $\varepsilon^{R O}$ is a very small positive number named the convergence threshold of the RO problem. And the final scheduling plan $y_{i}^{l+1}$ will be regarded as the robust scheduling plan considering the worst scenario of renewable energy.

\section{Distributed Robust Model Predictive Control- BASED ENERGy MANAGEMENT STRATEGY FOR MulTi- MICROGRIDS}

\section{A. Dynamic Alliance for Energy Trading}

As shown in Fig. 1, each MG has its own information server, and the server contains a buyer aggregator (BA) and a seller aggregator (SA). The one which is allowed to be called depends on the identity of the microgrid in the electricity market at the current time. That means each aggregator represents the behavior of sellers and buyers in the strategy.

The same aggregator unifies information among MGs. In order to transmit the minimum necessary information to ensure the privacy of the operating conditions, only the bidding power and power prices of the buyers and sellers are allowed to be transmitted in networks.

In each sampling point in the prediction layer, operators show their identity to the corresponding aggregator according to their own needs. The seller and buyer alliances will be formed dynamically before closing the power market. And each alliance 


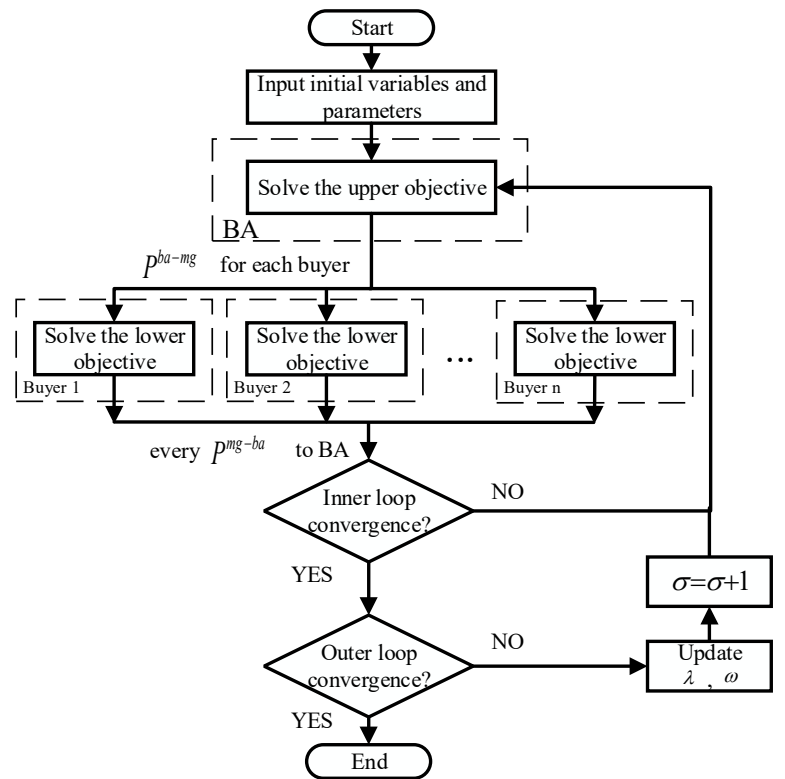

Fig. 4 The ATC-based strategy for buyer alliance.

has its own strategy which forming a flexible power market in which cooperation and non-cooperation coexist. In order to mobilize the enthusiasm of members in the microgrid cluster to participate in power market, the energy finally sold to the market when the microgrid acts as a seller is regarded as the contribution of itself to the cluster [24].

\section{B. Strategy for Seller Aggregator}

For long-term operations in a microgrid cluster based on the same cooperation agreement, the accumulation of individual contributions to the group can greatly affect their priority in the power market. For sellers, selling renewable energy to demand users is the only way to accumulate their own contribution. Therefore, sellers want to maximize their own contribution while ensuring their own interests. That is to say, there is a competitive relationship among sellers. The contribution factor of sellers is calculated as follows:

$$
L_{i}^{S}(k)=\sum_{i=1}^{k} L_{i, \mathrm{t}} / L_{\text {sum }}
$$

where $L_{i, \mathrm{t}}$ is the contribution of MG to the cluster as a seller at every moment in the past. $L_{\text {sum }}$ is the total contribution value accumulated by all MGs. And as a way to increase income and enthusiasm of sellers for selling excess energy, the bidding price is related to seller contribution factor:

$$
\omega_{i}^{\text {bid }}(k)=\omega_{i}^{\text {bas }}+\sigma \cdot L_{i}^{S}(k)
$$

where $\omega_{i}^{b a s}$ is the basic price to ensure basic income. $\sigma$ is unified to all microgrids which indicates the degree of influence of the contribution factor on price. And the final bidding price $\omega^{\text {tra }}$ from seller alliance is the average price which is calculated by

$$
\begin{gathered}
\omega^{\text {tra }}(k)=\frac{\sum_{m} P_{i}^{\text {sug }}(k) \cdot \omega_{i}^{\text {bid }}(k)}{\sum_{m} P_{i}^{\text {sug }}(k)} \\
S(k)=\sum_{i} P_{i}^{\text {sug }}(k)
\end{gathered}
$$

where $P_{i}^{\text {sug }}$ indicates the bidding power from $\mathrm{MG} i$. And $P_{i}^{\text {sug }}=0$ when it wants to be a buyer. $S$ is total bidding power. And the objective function of sellers can be rewritten as:

$$
\Phi_{\text {seller }}=\sum_{k=1}^{M}\left\{\min _{P_{i, k}^{s u g}} \omega_{k}^{\text {tra }}\left(P_{k}^{\text {ba-mg }}-P_{i, k}^{\text {sug }}\right)+\max _{U} \min _{\Omega} f_{i}^{T} \cdot y_{i}\right\}
$$

where $P_{k}^{b a-m g}$ is the purchased energy, which will be discussed in Section III-C.

From the overall point of view of the strategy, uncertain natural resources are regarded as virtual players to play a noncooperative game with microgrid. On the other hand, sellers also need to game with buyers to make trade-offs between their own profits and accumulated contributions.

\section{Strategy for Buyer Aggregator}

For BA, it is necessary to strive for the minimum cost from the sellers, but also to maintain the principle of fairness to the internal members. Therefore, a cooperative approach based on the ATC method is taken into account in the buyer aggregator.

In the process of allocating resources in an alliance based on the contribution, it is necessary to the priority of the buyer's harvest considers the current demand ratio and accumulated contribution value. However, compared with the contribution factor for sellers that can be quickly accumulated, a calculation method for the contribution factor of buyers of diminishing influence over time is used to further ensure the fairness of the transaction and prevent the occurrence of monopoly.

$$
L_{i}^{B}(k)=\frac{\sum_{t}^{k}\left(L_{i} \cdot(1 /(k-t+1))\right.}{L^{\text {sum }}} \quad t=1,2, \cdots, k
$$

And the priority factor $\mu_{i}$ of buyers is calculated as follows:

$$
\mu_{i}(k)=\frac{d_{i}(k)}{D(k)}+\pi \cdot L_{i}^{B}(k)
$$

where $d_{i}$ and $D$ are power demand of MG $i$ and total demand of buyer alliance, respectively; $\pi$ is the contribution coefficient.

As shown in Fig. 4, the strategy for buyer alliance based on ATC method is divided into two stages. On the upper level, the BA needs to consider the maximization of overall expectation of all buyers to allocate the purchased energy and minimization of cost of purchasing energy from sellers. The objective function is shown as:

$$
\begin{gathered}
\Phi_{b a}=\min \left(\alpha \cdot \Phi_{1}+\gamma \cdot \Phi_{3}\right)+\max \beta \cdot \Phi_{2} \\
\left\{\begin{array}{c}
\Phi_{1}=\sum_{k} \omega^{t r a}(k) \cdot P^{s}(k) \cdot \Delta T \\
\Phi_{2}=\sum_{i} \sum_{k} \mu_{i}(k) \cdot \log \left(1+P_{i, \sigma}^{b a-m g}(k) / P_{i, \sigma-1}^{m g-b a}(k)\right) \\
\Phi_{3}=\sum_{i} \sum_{k}\left[\lambda_{i}^{\sigma}(k) \cdot\left(P_{i, \sigma}^{b a-m g}(k)-P_{i, \sigma-1}^{m g-b a}(k)\right)\right. \\
\left.+\omega_{i}^{\sigma}(k)^{2} \cdot\left(P_{i, \sigma}^{b a-m g}(k)-P_{i, \sigma-1}^{m g-b a}(k)\right)^{2}\right] \\
0 \leq P^{s}(k) \leq S(k) \\
P^{s}(k)=\sum_{i} P_{i, \sigma}^{b a-m g}(k)
\end{array}\right.
\end{gathered}
$$

$\Phi_{1}$ is the total cost of purchasing electricity from SA. $\Phi_{2}$ indicates the total utility of the buyers [25], and the purpose is to maximize the total benefits of all buyers based on the accumulation of contribution. $\Phi_{3}$ is the area coupling constraint of BA in ATC method, which aims to converge the expected power distribution from buyers and BA. Moreover, $\alpha, \beta$ and $\gamma$ are penalty coefficients to balance the proportion of each objective. $P^{s}(k)$ is the expected purchase amount of power from SA. $P_{i}^{b a-m g}$ and $P_{i}^{m g-b a}$ are optimal allocation to MG from BA 


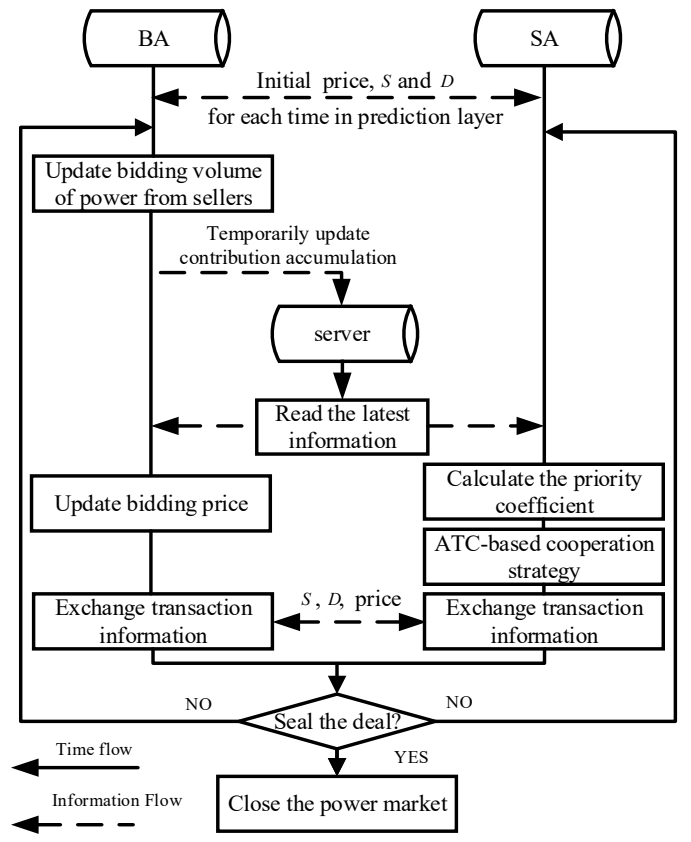

Fig. 5. Schematic diagram of the alliance-based power market.

and expected demand from MGs. $l l$ is the number of iterations. And $\lambda, \omega_{i}$ are LaGrange multiplier and penalty factor.

On the lower level, MGs only need to consider two aspects of the goal, its own cost and different opinion from the allocation of BA.

$$
\begin{gathered}
\Phi_{b u y e r}=\min _{P_{i, \sigma}^{m g-b a}}\left(\Phi_{4}+v \cdot \Phi_{5}\right) \\
\left\{\begin{array}{c}
\Phi_{4}=\sum_{k=1}^{M}\left\{\omega^{\text {tra }}(k) \cdot\left(P_{i, l l}^{m g-b a}(k)-P_{i}^{s u g}(k)\right)+f_{i}^{T} \cdot y_{i}\right\} \\
\Phi_{5}=\sum_{k}\left[\lambda_{i}(k) \cdot\left(P_{i, l l}^{m g-b a}(k)-P_{i, l l-1}^{b a-m g}(k)\right)\right. \\
\left.+\omega_{i}(k)^{2} \cdot\left(P_{i, l l}^{m g-b a}(k)-P_{i, l l-1}^{b a-m g}(k)\right)^{2}\right]
\end{array}\right.
\end{gathered}
$$

$\Phi_{4}$ is the cost of buyer. $\Phi_{5}$ is the area coupling constraint of MG similar to $\Phi_{3}$. And $v$ indicates the degree of compromise of the buyer to the alliance. And the convergence criterion and coefficient update are shown in (38) - (40).

$$
\begin{aligned}
& \max \left[\frac{\left|\Phi_{b a}^{l l}-\Phi_{b a}^{l l-1}\right|}{\left|\Phi_{b a}^{l l}\right|+1}, \frac{\left|\Phi_{\text {buyer }}^{l l}-\Phi_{\text {buyer }}^{l l-1}\right|}{\left|\Phi_{\text {buyer }}^{l l}\right|+1}\right] \leq \varepsilon^{\text {inner }} \\
& \int\left\|\sum_{k}\left(P_{i, l l}^{b a-m g}-P_{i, l l}^{m g-b a}\right)\right\|_{\infty} \leq \varepsilon^{\text {outer }} \\
& \left\{\left\|\sum_{k}\left(P_{i, l l}^{b a-m g}-P_{i, l l}^{m g-b a}-\left(P_{i, l l-1}^{b a-m g}-P_{i, l l-1}^{m g-b a}\right)\right)\right\|_{\infty} \leq \varepsilon^{\text {outer }}\right. \\
& \left\{\lambda_{i}^{l l+1}(k)=\lambda_{i}^{l l}(k)+2\left(\omega_{i}^{l l}(k) \circ \omega_{i}^{l l}(k)\right) \circ\left(\sum_{k}\left(P_{i, l l}^{b a-m g}-P_{i, l l}^{m g-b a}\right)\right)\right. \\
& \omega_{i}^{l l+1}(k)=\kappa \cdot \omega_{i}^{l l}(k)
\end{aligned}
$$

Equation (38) is the converged condition of the inner loop and Equation (39) is of the outer loop. And the convergence coefficient $\lambda, \omega_{i}$ will be updated according to Equation (40) if the outer loop does not converge.

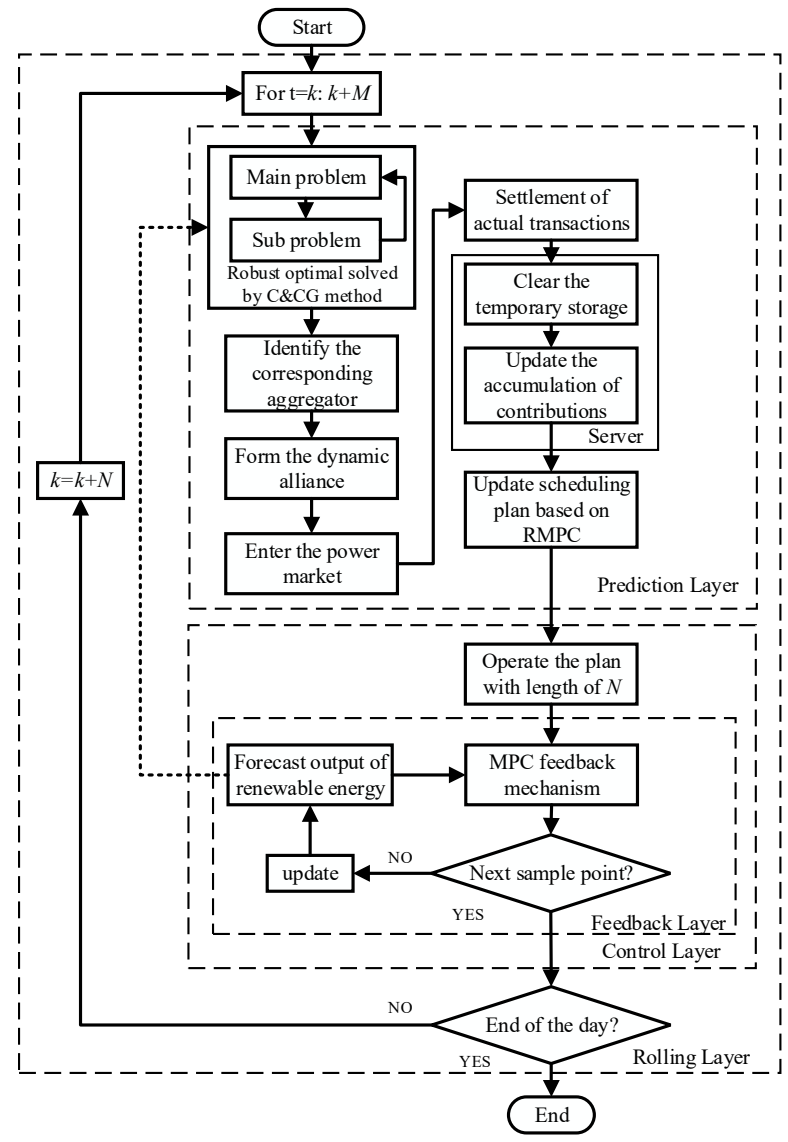

Fig. 6. DMPC-based energy management strategy for multi-microgrids.

And finally, when the opinions are unified (convergence is complete), $P^{b a-m g}$ will be used as the decision amount for BA to distribute the purchased energy to buyers.

\section{Distributed Robust Model Predictive Control-Based Energy Management Strategy}

After obtaining the optimal scheduling plan calculated by RMPC prediction module mentioned in Section II, operators will show their identity in each sampling point to BA or SA according to their wishes to be a buyer or seller. And after the formation of the dynamic trading alliance, power market trading will be opened.

After exchanging the expected transaction volume ( $S$ and $D$ ) and broadcast to the corresponding demanders, the two alliances will act separately according to the strategies we mentioned in Sections III-B and C. For BA, only the expected allocation of power $\left(b u=\left\{P_{i}^{b a-m g}, P_{i}^{m g-b a}\right\}\right)$ can be exchanged between BA and buyers, which will maximize the privacy of microgrid information. The same for $\mathrm{SA}, s e=\left[P_{i}^{s u g}, \omega_{i}^{\text {bid }}\right]$ is the only circulating bidding information including power $\left(P_{i}^{\text {sug }}\right)$ and price $\left(\omega_{i}^{\text {bid }}\right)$. Therefore, the information that each microgrid needs to upload to the aggregator can be summarized as

$$
\left\{\begin{array}{l}
P_{i, \mathrm{t}}^{m g-a g g}=\left[i d_{i}^{t}, \text { mar }_{i}^{t}\right] \quad t=1,2 \ldots k \\
\operatorname{mar}_{i}^{t}= \begin{cases}b u_{i}^{t} & \text { if } \mathrm{MG} \text { is a buyer } \\
s e_{i}^{t} & \text { if } \mathrm{MG} \text { is a seller }\end{cases}
\end{array}\right.
$$


This article has been accepted for publication in a future issue of this journal, but has not been fully edited. Content may change prior to final publication. Citation information: DOI10.1109/TSG.2022.3147370,

IEEE TRANSACTIONS ON SMART GRID, VOL. XX, NO. XX, XXX 20XX

TABLE I

MICROGRIDS PARAMETERS

\begin{tabular}{ccccccc}
\hline \hline & MG 1 & MG 2 & MG 3 & MG 4 & MG 5 & MG 6 \\
\hline PV $(\mathrm{kW})$ & 700 & 800 & 700 & 500 & 600 & 500 \\
WT $(\mathrm{kW})$ & 1200 & 1000 & 1300 & 1300 & 1200 & 1200 \\
BESS $(\mathrm{kWh})$ & 4000 & 4000 & 5000 & 5500 & 4500 & 6000 \\
PG $(\mathrm{kW})$ & 800 & 800 & 800 & 800 & 800 & 800 \\
\hline \hline
\end{tabular}

TABLE II

OPERATION PARAMETERS

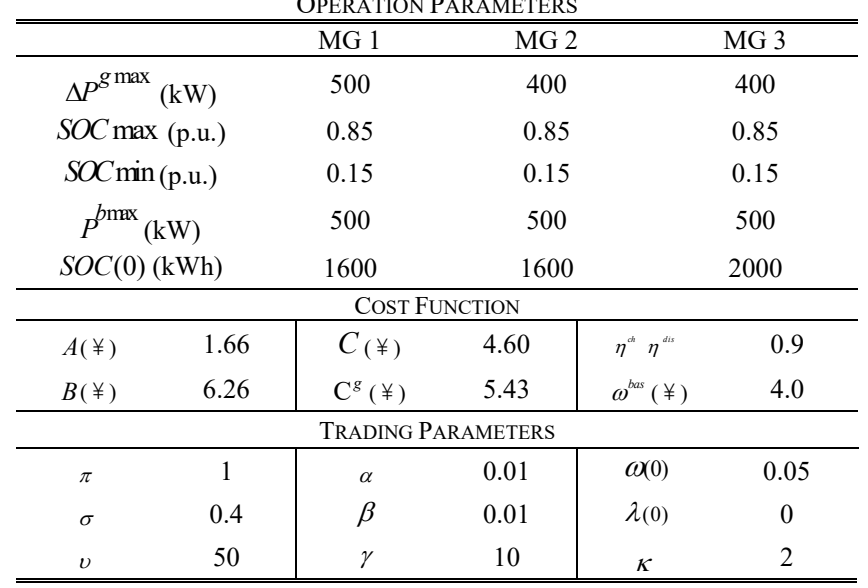

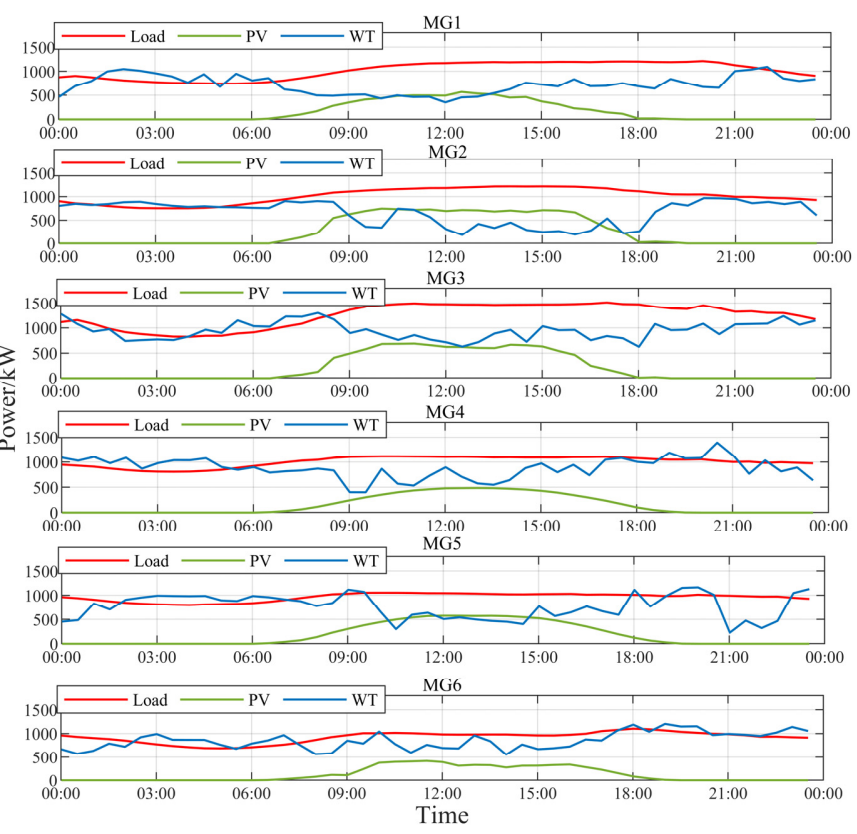

Fig. 7. The forecast curves of renewable energy and loads.

In addition, for SA, the sales share will be compulsorily distributed to each seller in proportion as:

$$
P_{i}^{\text {sug }}=\frac{D(k) \cdot P_{i}^{\text {sex }}}{\sum P_{i}^{\text {sex }}}
$$

where $P_{i}^{\mathrm{sex}}$ is the initial bidding volume of power from sellers.

Therefore, the response information which the aggregator returns to each microgrid will be sent as

$$
P M_{i, t}^{a g g-m g}=\left\{\begin{array}{l}
P_{i, k}^{b a-m g} \quad \text { from BA to buyer } \\
{\left[\omega_{t}^{t r a}, P_{i, t}^{\text {sug }}\right] \text { from SA to seller }}
\end{array} \quad t=1,2 \ldots k\right.
$$
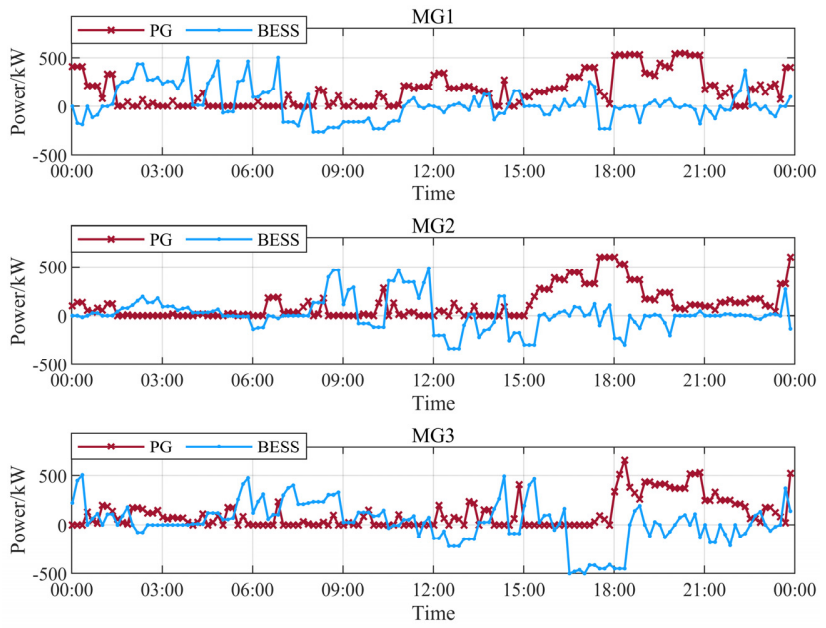

Fig. 8. Scheduling plan of three MGs controlled by RMPC strategy.

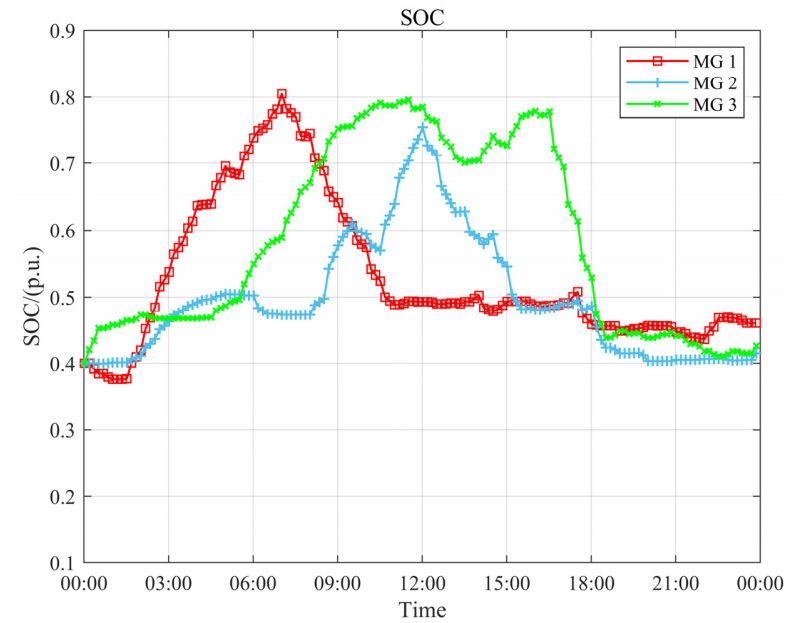

Fig. 9. Changes of SOC with RMPC strategy intraday.

Then the lasted bidding information will be exchanged until the consensus in the market between BA and SA. The demand from two alliances is updated as (28) and (44).

The schematic diagram of the alliance-based power market is shown in Fig. 5.

$$
D(k)=P^{S}(k)
$$

While all the transactions are finished satisfactorily, it should be noted that, only the transaction included in the control amount of length $N$ can be truly implemented. The latest accumulation of contributions will be updated in the server based on actual transactions.

Finally, the MGs will re-plan the scheduling based on the transaction information. And then, the feedback mechanism will be operated independently in each microgrid until the next time of power market opens. The whole DMPC-based energy management strategy for multi-microgrids is shown in Fig. 6.

\section{VERIFICATION RESULTS AND DisCUSSIONS}

As mentioned in Section II-B, each MG in the island shown in Fig. 1 can be controlled by the RMPC strategy. The forecast curves of the renewable energy and loads with 30-minute intervals of six islanded interconnected MGs on island group are shown in Fig. 7. Each curve of renewable energy is predicted by a neural network mentioned in Section II-C with 20 days of real 

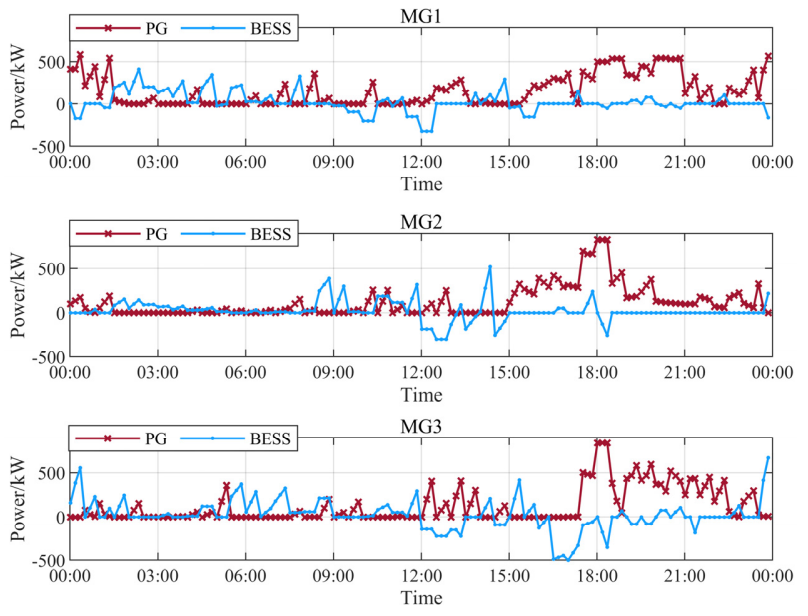

Fig. 10. Scheduling plan of three MGs controlled by DRMPC strategy.

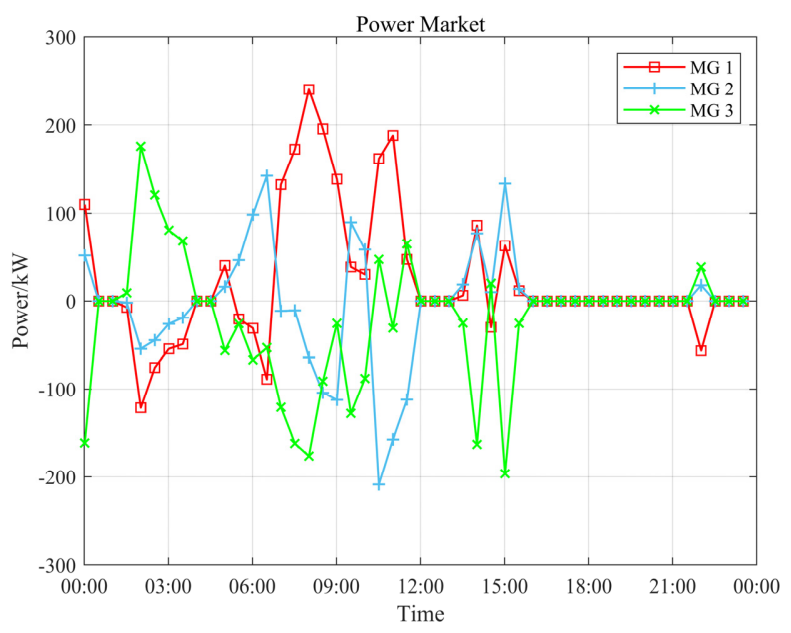

Fig. 11. Transactions in the power market intraday among 3 MGs.

historical data. We set the rolling layer $P=48$, the prediction layer $M=8$, and the control layer $N=1$. This means that the sampling interval for rolling layer is 30 -minute and 10-minute for feedback layer, the time scale of the prediction layer is 4 hours.

It should be noted that, in order to facilitate the analysis and explanation, only three microgrids are simulated in Sections IVA to $\mathrm{D}$ to show the feasibility of the proposed strategy. The extended discussion for more microgrids is presented in Section IV-E. In addition, the construction configuration parameters and operating parameters of the microgrids are listed in Tables I and II, respectively.

\section{A. RMPC Strategy Applied in Independent Microgrid}

The scheduling plan of three independent microgrids based on the RMPC strategy is shown in Fig. 8, in which the uncertainty factor $\Gamma=4$ and the allowable range of predictive error in Equation (1) are all 5\%. Each microgrid achieves the balance of supply and demand based on its own forecast data, and also can operate independently without connecting with others. The significance of this is that in the complex and changeable environment of the islands, the independent microgrid needs to face communication interruption or reduce mutual power transmission to ensure power quality at any time.

As shown in Figs. 7 and 8, due to the geographical proximity, the daily fluctuation trends of the renewable energy of the three

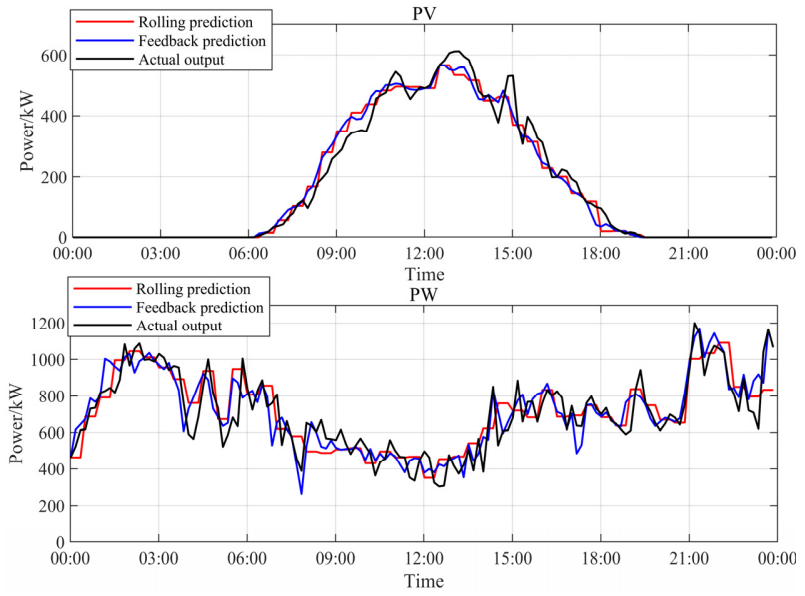

Fig. 12. The prediction output of renewable energy of MG 1. (a) PV. (b) WT.

MGs are similar. However, because of the different configurations and load levels, the surplus and deficit levels of three within a day are different. For example, from 0 A.M. to 8 A.M., MG 1 and MG 3 have some energy storage behavior, which results in using BESS as much as possible to meet demand between 8 A.M. and 10 A.M. However, the three microgrids have different degrees of power shortage from 3 P.M. to 21 P.M.

In addition, the SOC change of BESS is shown in Fig. 9. Except for the time of extremely abundant renewable energy, the BESS fluctuates but maintains at a very healthy operating level, which is what we expect from (18).

\section{B. DRMPC-based Energy Management Strategy}

The proposed DRMPC strategy in this paper is adopted in operation, and the results are shown in Fig. 10. On the whole, the operating range of the power generation units of each microgrid is effectively reduced within a day, especially in the morning and noon. This is the benefit brought by the power transaction among MGs. However, the improvement of the operation of the three MGs is limited in the evening, since the wind power in the entire region shows a downward trend and lack of PV power generation when sunlight is insufficient. It is also worth noting that there are still many fluctuating spikes in the expected scheduling plan. This is caused by the feedback mechanism of their own operation. Therefore, the adjustment pressure will still be caused on the equipment in pursuit of economic efficiency. Opening up a free market in a shorter period may be a way to further pursue operational stability and economic efficiency [31].

As shown in Fig. 11, the flexible transactions and fair distribution are realized among microgrids during the day. The MG represents the identity of a seller when the power is less than zero. Under the strategy based on contribution accumulation, MGs are effectively motivated to invest as much energy trading as possible in the proposed power market. The contribution accumulation is more important for players who are buyers. Because when they become a buyer, they will get a proportion of energy distribution with priority based on contribution proportion. As we have analyzed in Fig. 10, the negative output of renewable energy from 4 P.M. to 21 P.M. also led to the closure of the electricity market, which will provide a reference for the long-term expansion of personal MG facilities to seize vacancies of the power market. However, it should be noted that it does not mean the negativity of the proposed framework of strategy, because of the decision made by operators to ensure the 

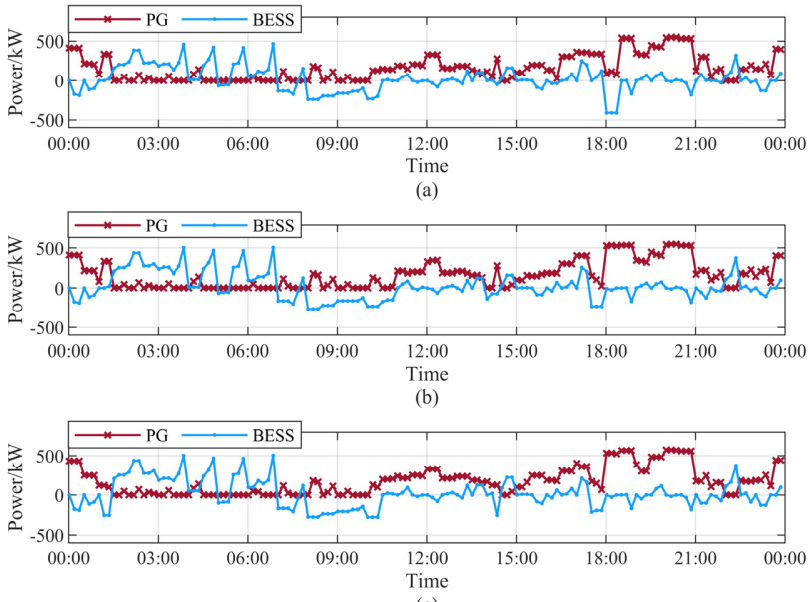

Fig. 13. Scheduling plan under different uncertainty factors for MG 1. (a) $\Gamma=0$ (b) $\Gamma=4$. (c) $\Gamma=8$.
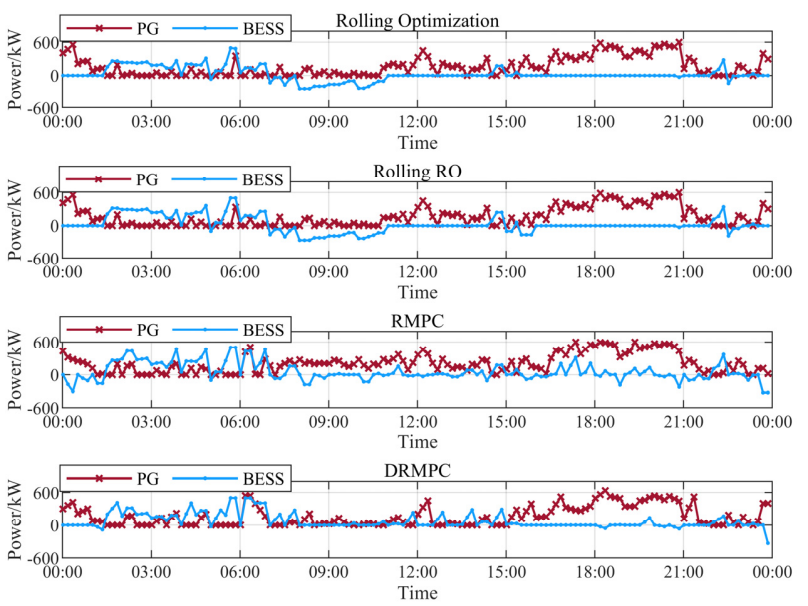

Fig. 14. Real operating conditions under different strategies for MG 1 .

stable operation of the microgrid by independent operating. With the addition of more microgrids, the market will become more active, which will be discussed in Section IV-E.

\section{Combating to the Uncertainty of Renewable Energy}

For the feedback mechanism in MPC, its ability to deal with the uncertainty of renewable energy depends first on the accuracy of its forecast correction. The forecast correction curve of the feedback module is shown in Fig. 12, in which the prediction curve has been updated in real time according to the historical and actual sampled data. This rolling prediction method based on historical and real-time data makes the correction curve in ultra-short timescale closer to the actual output situation, so that the output error can be corrected in advance in the correction module, which effectively distributes the adjustment pressure on the generator and improves the safety margin of the microgrid operation [29].

Due to the adjustability of the uncertainty factor, the worst scenario for a short period can be considered. Compared to the MPC strategy, the RMPC strategy including RO can help the system respond to the uncertainty of renewable energy one step ahead of time. Taking MG 1 with RMPC strategy as an example, the scheduling plans with different uncertainty factors are shown in Fig. 13. It can be seen from the comparison that with the increase of the uncertainty factor, the transfer of the controllable
TABLE III

AVERAGE RELATIVE ERROR OF THE NET LOAD

\begin{tabular}{cccc}
\hline \hline Operating Strategies & MG 1 & MG 2 & MG 3 \\
\hline RMPC $(\Gamma=4)$ & 2.0492 & 2.6362 & 3.9265 \\
Distribution rolling & 0.9465 & 1.3860 & 1.8240 \\
optimization & 0.8711 & 1.1620 & 1.6166 \\
DMPC & 0.9785 & 1.5123 & 2.0114 \\
DRMPC $(\Gamma=4)$ & &
\end{tabular}

TABLE IV

OPERATING COST OF THREE STRATEGIES $\left(\times 10^{4} ¥\right)$

\begin{tabular}{cccc}
\hline \hline Operating Strategies & MG 1 & MG 2 & MG 3 \\
\hline MPC & 2.623 & 1.815 & 2.296 \\
$\operatorname{RMPC}(\Gamma=4)$ & 2.809 & 2.159 & 2.370 \\
$\operatorname{DMPC}$ & 2.531 & 1.738 & 2.184 \\
DRMPC $(\Gamma=4)$ & 2.660 & 1.966 & 2.206 \\
\hline \hline
\end{tabular}

power generation equipment becomes more stable. That is because of responding to the worst scenarios in advance by robust optimization, the pressure on devices that need to be adjusted quickly in the feedback mechanism will reduce, which can make the operation of the microgrid more stable.

Moreover, the average relative error of the net load is shown in Table III [32]. The calculated result of error with the DMPC strategy is better than the rolling optimization which is fully pursuing the economy. That is because the prediction curve is closer to the actual output curve by using the feedback mechanism. And because of the same predictive model used in the three MGs, the improvement effect is similar. However, comparing the DRMPC strategy with the DMPC strategy, this value has risen slightly. This is because the worst scenario predicted by the RO is not based on real data but based on the operating status of the device. Therefore, it does not necessarily match the real scenario in actual operation, which may cause more operational errors. But for operators facing uncertain factors in the future operation of $\mathrm{MG}$, such errors can be accepted in exchange for preventing the emergence of scenarios that disrupt the stable operation of the microgrid.

And the comparison of real operating conditions under different strategies is shown in Fig. 14. As we have analyzed before, compared with rolling optimization, the risk in the rolling RO strategy is allocated to all devices in advance. And as a further improvement strategy, RMPC uses a more accurate forecast curve to allocate risks more reasonably in the ultra-short term. For the same to have been shown in DRMPC strategy, in which the use of equipment is reduced on the basis of the DMPC strategy.

\section{Balance of Economy and Robustness}

RO and MPC strategies deal with the uncertainty of renewable in different time scales, which will correspondingly pay the price of economic loss for this. The comparison of the cost of intraday operation with different operating strategies is shown in Table IV.

Compared to RMPC strategy, MPC strategy pursues the minimization of cost in the short-term planning stage, so that the total cost can be controlled to be lower. This is because the uncertainty set of $\mathrm{RO}$ has the probability of violating the constraints. In order to ensure the feasible solution of strategy within the uncertainty range of renewable energy, the result of $\mathrm{RO}$ is a conservative solution. It is called 'The Price of 


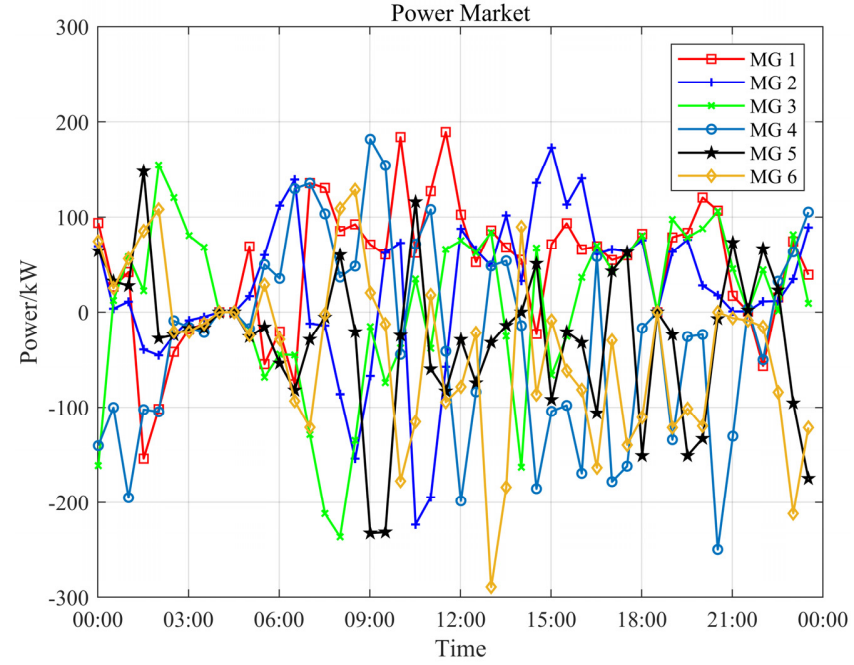

Fig. 15. Transactions in the power market intraday among 6 MGs.

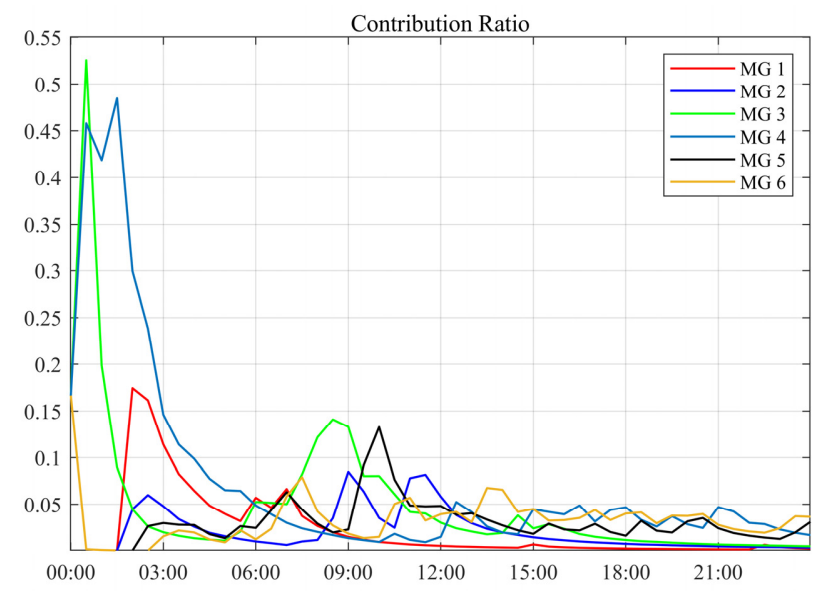

Fig. 16. Real-time contribution ratio among buyers.

Robustness', which is directly reflected on the changes of economic cost [3]. As shown in Table IV, the larger the $\Gamma$, the more conservative the robust solution, and the greater the price (extra cost) paid. Therefore, robustness and economy are negatively correlated. That is, when only the uncertainty parameter $\Gamma$ changes, the change in cost reflects the degree of the conservativeness of the robust solution. However, the proposed free power market framework constructs a trading framework and enhances the enthusiasm for interaction among MGs, which can reduce the 'Price' that operators need to pay extra. Therefore, based on the objective of RMPC to enhance the robustness of the system, the proposed DRMPC strategy further reduces the operating cost of each microgrid. Compared with the independent operation, the daily operating cost of each MG with the DRMPC strategy has been reduced by $5.3 \%, 8.9 \%$ and $6.9 \%$.

And if the importance of contribution accumulation in the strategy is further increased to stimulate transactions among microgrids, the cost may be further reduced. But as we analyzed in Section IV-C, it depends on operators to weigh the balance between risks and benefits. As a whole, the proposed DRMPC strategy has the ability to be easily adjusted whether it is operated independently or with networked interaction, which depends on the expectations of the operators and the agreement with other members. Therefore, the proposed DRMPC strategy can well balance the robustness and economy of microgrid operation,
TABLE V

ENERGY ALLOCATED FOR BUYERS USED DIFFERENT METHOD

\begin{tabular}{|c|c|c|c|c|}
\hline Time & \multicolumn{4}{|c|}{ 7:30 A.M. } \\
\hline Buyer & \multicolumn{2}{|c|}{ MG $1(\mathrm{~kW})$} & \multicolumn{2}{|c|}{ MG $4(\mathrm{~kW})$} \\
\hline Demand & \multicolumn{2}{|c|}{172.46} & \multicolumn{2}{|c|}{132.27} \\
\hline Method in [33] & \multicolumn{2}{|c|}{116.84} & \multicolumn{2}{|c|}{116.84} \\
\hline Method in [19] & \multicolumn{2}{|c|}{136.93} & \multicolumn{2}{|c|}{96.75} \\
\hline Method in [25] & \multicolumn{2}{|c|}{145.7} & \multicolumn{2}{|c|}{87.97} \\
\hline This paper & \multicolumn{2}{|c|}{130.42} & \multicolumn{2}{|c|}{103.26} \\
\hline Time & \multicolumn{4}{|c|}{ 8:00 A.M. } \\
\hline Buyer & $\begin{array}{l}\text { MG } 1 \\
(\mathrm{~kW})\end{array}$ & $\begin{array}{c}\text { MG } 4 \\
(\mathrm{~kW})\end{array}$ & $\begin{array}{l}\text { MG 5 } \\
(\mathrm{kW})\end{array}$ & $\begin{array}{c}\text { MG 6 } \\
(\mathrm{kW})\end{array}$ \\
\hline Demand & 240.4146 & 61.0483 & 71.0326 & 222.9883 \\
\hline Method in [33] & 80.54 & 80.54 & 80.54 & 80.54 \\
\hline Method in [19] & 169.65 & 0 & 0 & 152.23 \\
\hline Method in [25] & 199.09 & 0 & 0 & 123.065 \\
\hline This paper & 105.28 & 38.51 & 49.53 & 119.80 \\
\hline
\end{tabular}

which all are valued most from operators.

\section{E. Expansion of the multi-microgrids system}

When the multi-microgrids system expands, the number of microgrids increases, and the performance of the electricity market will be more active. Taking the expansion to six microgrids as an example, the results of liquidation in the power market is shown in Fig. 15, in which all microgrids participate with the proposed DRMPC strategy. Compared with the smallscale markets of the three microgrids in Section IV-B, there are more transactions and transaction options among microgrids. As shown in Fig. 15, the trading behavior basically covers the whole day. And compared with Fig. 11, there are fewer market vacancies. This is because more participants provide more market uncertainty, which is a necessary condition for the market to be active.

The result of the contribution calculation method given in Equation (30) is shown in Fig. 16. The contribution of MGs as a seller will increase the allocation priority when becoming a buyer, but will gradually diminish over time if there is no continuous contribution. For example, between 0 A.M. and 2 A.M., due to the large surplus of renewable energy, the selling behaviors of MG 3 and MG 4 make them basically occupied the contribution accumulation. If the contribution rate is only calculated by accumulation [25], the two microgrids will gain a great advantage in allocated energy as a buyer when other microgrids are unable to sell in large quantities for a period of time. However, from the results of the contribution accumulation method in this paper, due to the lack of continuous contributions from these two microgrids, the time to enjoy the advantages is also limited, which has avoided this kind of monopoly. As shown in Fig. 16, when all microgrids begin to be active in the market as sellers during the day, the contribution rate begins to show a trend of fair competition.

However, as a simulation of an independent day, it is inevitable that the contribution rate shows a common downward trend. If the proposed DRMPC strategy is applied to the longterm operation of the multi-microgrids system, this problem can be solved by adjusting the rate of decline of contribution degree and the proportion of contribution degree in the priority factor in Equations (30) and (31), in which the prerequisite is the consent of all participants.

In addition, in order to show more details, the allocated energy results compare with the methods in other references for buyers 
This article has been accepted for publication in a future issue of this journal, but has not been fully edited. Content may change prior to final publication. Citation information: DOI10.1109/TSG.2022.3147370,

at 7:30 A.M. and 8:00 A.M. are shown in Table V. For the method in [33], each buyer will receive the same allocation. In [18], the goal of allocation is to maximize the utilization rate of renewable energy, that is, to minimize the sum of squares of the deviation between the allocation and demand. The objective of the method in [25] is to maximize social welfare, and the calculation method of contribution ratio in buyer priority factor is the proportion of self-contribution accumulation in the total contribution.

The method in [33] allows every buyer to equally share energy that ignores the actual demand. In this way, as shown at 8 A.M., MG 1 and MG 6 with higher demand are allocated insufficient energy, while MG 4 and MG 5 with lower demand have obtained excessive energy which possibly unnecessary. The method in [19] maximizes the use of renewable energy, that is, to take into account the demand ratio. However, as shown at 8 A.M., the microgrids such as MG 4 and MG 5 with smaller demand are ignored in the allocation plan, which is unfair. Although these two methods have cooperative optimization schemes, the allocation plan for buyers has nothing to do with historical behavior. Some microgrids will make great sacrifices for the cluster which will reduce the enthusiasm and willingness to contribute energy to the cluster; thus, it is unable to establish an attractive free power market. For the method in [25], compared to MG 1 at the two moments, a higher proportion of energy is allocated. This is because the energy that sold at 1:30 A.M. will lead to an absolute dominance in the early morning, and will still continue for some time in the future. This is detrimental to the construction of a competitive market. And as shown at 8 A.M., the smaller demand from MG 4 and MG 5 also have been ignored by aiming at the maximization of the social welfare, even though MG 4 has contributed a lot to the cluster in the past.

In comparison, the influence of MG 1 contribution in the past has gradually weakened in these two time periods by the proposed DRMPC strategy. And even the gap among the demand from buyers is large, the results of allocation are still reasonable. The fairer result will in turn stimulate the microgrid to actively participate in the power market. This effectively builds a market environment for continuous competition, which is very helpful for the long-term operation of the multimicrogrids system with multiple owners.

\section{CONCLUSION}

In this paper, a distributed robust model predictive control strategy is proposed to optimize energy management for multimicrogrids considering uncertainty. This strategy firstly guarantees the stable operation of the independent microgrid with RMPC strategy, in which the uncertainty of renewable energy can be mitigated by using the robust optimization and the feedback mechanism of MPC on different time scales. These two mechanisms complement each other which improve the robustness of microgrid operations and the safety of equipment operations at the expense of part of the economy. However, due to the adjustment of multiple operating parameters, operators can balance robustness and economy more flexibly. Furthermore, each member in multi-microgrids can form a dynamic trading alliance according to their own needs, and achieve a free power market framework based on community contribution in which cooperation and non-cooperation coexist with different goals.
This is more in line with the complex market environment and the psychological game of operators. The proposed distributed control strategy with minimum necessary information interaction effectively guarantees the privacy of microgrid operation information.

\section{REFERENCES}

[1] M. F. Zia, E. Elbouchikhi, and M. Benbouzid, "Microgrids energy management systems: A critical review on methods, solutions, and prospects," Applied Energy, vol. 222, pp. 1033-1055, Jul. 2018.

[2] B. Chen, J. Wang, X. Lu, C. Chen, and S. Zhao, "Networked microgrids for grid resilience, robustness, and efficiency: A review," IEEE Trans. Smart Grid, vol. 12, no. 1, pp. 18-32, Jan. 2021

[3] D. Bertsimas and M. Sim, "The price of robustness," Operations Research, vol. 52, no. 1, pp. 35-53, Feb. 2004.

[4] Y. Xiang, J. Liu, and Y. Liu, "Robust energy management of microgrid with uncertain renewable generation and load," IEEE Trans. Smart Grid, pp. 1-1, 2015.

[5] G. Liu, Y. Xu, and K. Tomsovic, "Bidding strategy for microgrid in dayahead market based on hybrid stochastic/robust optimization," IEEE Trans. Smart Grid, vol. 7, no. 1, pp. 227-237, Jan. 2016.

[6] C. Zhang, Y. Xu, Z. Y. Dong, and L. F. Yang, "Multitimescale coordinated adaptive robust operation for industrial multienergy microgrids with load allocation," IEEE Trans. Ind. Informat., vol. 16, no. 5, pp.3051-3063, May 2020

[7] M. Nazari-Heris, B. Mohammadi-Ivatloo, G. B. Gharehpetian, and M. Shahidehpour, "Robust short-term scheduling of integrated heat and power microgrids," IEEE Systems Journal, vol. 13, no. 3, pp. 3295-3303, Sep. 2019.

[8] A. Parisio, E. Rikos, and L. Glielmo, "A model predictive control approach to microgrid operation optimization," IEEE Trans. Contr. Syst. Technol., vol. 22, no. 5, pp. 1813-1827, Sep. 2014.

[9] Y. Li, X. Fan, Z. Cai and B. Yu, "Optimal active power dispatching of microgrid and distribution network based on model predictive control," in Tsinghua Science and Technology, vol. 23, no. 3, pp. 266-276, June 2018.

[10] P. Kou, D. Liang, L. Gao and F. Gao, "Stochastic coordination of plug-in electric vehicles and wind turbines in microgrid: a model predictive control approach," IEEE Trans. Smart Grid, vol. 7, no. 3, pp. 1537-1551, May 2016.

[11] M. A. Velasquez, J. Barreiro-Gomez, N. Quijano, A. I. Cadena, and M. Shahidehpour, "Intra-hour microgrid economic dispatch based on model predictive control," IEEE Trans. Smart Grid, pp. 1-1, 2019

[12] C. Wu, S. Gao, Y. Liu, T. E. Song, and H. Han, "A model predictive control approach in microgrid considering multi-uncertainty of electric vehicles,' Renewable Energy, vol. 163, pp. 1385-1396, Jan. 2021

[13] S. Cai, Y. Xie, Q. Wu, and Z. Xiang, "Robust MPC-based microgrid scheduling for resilience enhancement of distribution system," International Journal of Electrical Power \& Energy Systems, vol. 121, p. 106068, Oct. 2020

[14] M. I. S. Lahanda Purage, A. Krishnan, Y. S. E. Foo, and H. B. Gooi, "Cooperative bidding-based robust optimal energy management of multimicrogrids," IEEE Trans. Ind. Informat., pp. 1-1, 2019.

[15] M. Mohiti, H. Monsef, A. Anvari-moghaddam, J. Guerrero, and H. Lesani, "A decentralized robust model for optimal operation of distribution companies with private microgrids," International Journal of Electrical Power \& Energy Systems, vol. 106, pp. 105-123, Mar. 2019.

[16] X. Zhou, Q. Ai, M. Yousif, "Two kinds of decentralized robust economic dispatch framework combined distribution network and multi-microgrids," Applied Energy, p. 16, 2019.

[17] N. Nikmehr, "Distributed robust operational optimization of networked microgrids embedded interconnected energy hubs," Energy, vol. 199, p. 117440 , May 2020.

[18] Y. Zheng, Y. Song, D. J. Hill, and K. Meng, "Online distributed mpc-based optimal scheduling for ev charging stations in distribution systems," IEEE Trans. Ind. Informat., vol. 15, no. 2, pp. 638-649, Feb. 2019.

[19] Y. Du, J. Wu, S. Li, C. Long, and I. Ch. Paschalidis, "Distributed mpc for coordinated energy efficiency utilization in microgrid systems," IEEE Trans. Smart Grid, vol. 10, no. 2, pp. 1781-1790, Mar. 2019.

[20] F. Garcia-Torres, C. Bordons, and M. A. Ridao, "Optimal economic schedule for a network of microgrids with hybrid energy storage system using distributed model predictive control," IEEE Trans. Ind. Electron., vol. 66, no. 3, pp. 1919-1929, Mar. 2019.

[21] Q. Zhang, K. Dehghanpour, Z. Wang, and Q. Huang, "A learning-based power management method for networked microgrids under incomplete information," IEEE Trans. Smart Grid, vol. 11, no. 2, pp. 1193-1204, Mar. 2020 . 
[22] Q. Zhang, K. Dehghanpour, Z. Wang, F. Qiu, and D. Zhao, "Multi-agent safe policy learning for power management of networked microgrids," IEEE Trans. Smart Grid, vol. 12, no. 2, pp. 1048-1062, Mar. 2021.

[23] Y. Liu, H. B. Gooi, Y. Li, H. Xin, and J. Ye, "A secure distributed transactive energy management scheme for multiple interconnected microgrids considering misbehaviors," IEEE Trans. Smart Grid, vol. 10, no. 6, pp. 5975-5986, Nov. 2019

[24] S. Park, J. Lee, S. Bae, G. Hwang, and J. K. Choi, "Contribution-based energy-trading mechanism in microgrids for future smart grid: a game theoretic approach," IEEE Trans. Ind. Electron., vol. 63, no. 7, pp. 42554265, Jul. 2016.

[25] A. M. Jadhav, N. R. Patne, and J. M. Guerrero, "A novel approach to neighborhood fair energy trading in a distribution network of multiple microgrid clusters," IEEE Trans. Ind. Electron., vol. 66, no. 2, pp. 1520 1531, Feb. 2019

[26] K. Dehghanpour and H. Nehrir, "An agent-based hierarchical bargaining framework for power management of multiple cooperative microgrids," IEEE Trans. Smart Grid, vol. 10, no. 1, pp. 514-522, Jan. 2019.

[27] L. An, J. Duan, M.-Y. Chow, and A. Duel-Hallen, "A distributed and resilient bargaining game for weather-predictive microgrid energy cooperation," IEEE Trans. Ind. Informat., vol. 15, no. 8, pp. 4721-4730, Aug. 2019.

[28] H. Wang and J. Huang, "Incentivizing energy trading for interconnected microgrids," IEEE Trans. Smart Grid, vol. 9, no. 4, pp. 2647-2657, Jul. 2018.

[29] Z. Zhao, J. Guo, C. S. Lai, H. Xiao, K. Zhou, and L. L. Lai, "Distributed model predictive control strategy for islands multi-microgrids based on non-cooperative game," IEEE Trans. Ind. Informat., pp. 1-1, 2020.

[30] R. A. Gupta and N. K. Gupta, "A robust optimization based approach for microgrid operation in deregulated environment," Energy Conversion and Management, vol. 93, pp. 121-131, Mar. 2015.

[31] F. Garcia-Torres, C. Bordons, J. Tobajas, J. J. Marquez, J. Garrido-Zafra, and A. Moreno-Munoz, "Optimal schedule for networked microgrids under deregulated power market environment using model predictive control," IEEE Trans. Smart Grid, vol. 12, no. 1, pp. 182-191, Jan. 2021.

[32] W. Gu, Z. Wang, Z. Wu, Z. Luo, Y. Tang and J. Wang, "An online optimal dispatch schedule for CCHP microgrids based on model predictive control," IEEE Trans. Smart Grid, vol. 8, no. 5, pp.2332-2342, Sept. 2017.

[33] J. Lee, J. Guo, J. K. Choi, and M. Zukerman, "Distributed energy trading in microgrids: a game-theoretic model and its equilibrium analysis," IEEE Trans. Ind. Electron., vol. 62, no. 6, pp. 3524-3533, Jun. 2015.

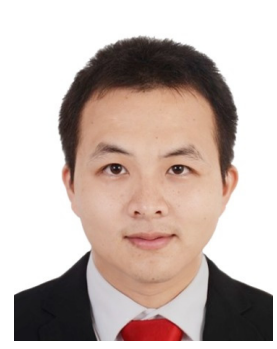

Zhuoli Zhao (S'15-M'18) received the $\mathrm{Ph} . \mathrm{D}$. degree in electrical engineering from South China University of Technology, Guangzhou, China, in 2017. From October 2014 to December 2015, he was a Joint Ph.D. Student and Sponsored Researcher with the Control and Power Research Group, Department of Electrical and Electronic Engineering, Imperial College London, London, U.K. He was a Research Associate with the Smart Grid Research Laboratory, Electric Power Research Institute, China Southern Power Grid, Guangzhou, China, from 2017 to 2018.

$\mathrm{He}$ is currently an Associate Professor with the School of Automation, Guangdong University of Technology, Guangzhou, China. His research interests include microgrid control and energy management, renewable power generation control and grid-connected operation, modeling, analysis and control of power-electronized power systems and smart grids. He is an Active Reviewer for the IEEE Transactions on Smart Grid, the IEEE Transactions on Sustainable Energy, the IEEE Transactions on Industrial Electronics, the IEEE Transactions on Power Electronics, and the Applied Energy.

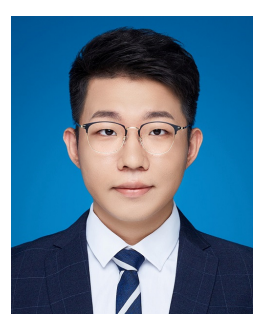

Juntao Guo received the B.E. degree from Guangdong University of Technology, Guangzhou, China, in 2019. He is currently working toward the master degree in electrical engineering with the School of Automation, Guangdong University of Technology, Guangzhou, China. His research interests include microgrid control and energy management.

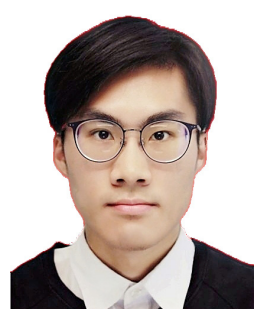

Xi Luo received the B.E. degree from Guangdong University of Technology, Guangzhou, China, in 2020. He is currently working toward the master degree in electrical engineering with the School of Automation, Guangdong University of Technology, Guangzhou, China. His research interests include microgrid control and power electronic converters.

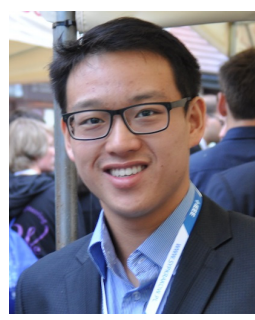

Chun Sing Lai (S'11, M'19, SM'20) received the B.Eng. (First Class Hons.) in electrical and electronic engineering from Brunel University London, London, UK, in 2013, and the D.Phil. degree in engineering science from the University of Oxford, Oxford, UK, in 2019.

$\mathrm{He}$ is currently a Lecturer with the Department of Electronic and Electrical Engineering, Brunel University London. From 2018 to 2020, he was an UK Engineering and Physical Sciences Research Council Research Fellow with the School of Civil Engineering, University of Leeds, Leeds, UK. His current research interests are in power system optimization and data analytics. Dr. Lai was the Publications Co-Chair for both 2020 and 2021 IEEE International Smart Cities Conferences. He is the Vice-Chair of the IEEE Smart Cities Publications Committee and Associate Editor for IET Energy Conversion and Economics. He is the Working Group Chair for IEEE P2814 Standard, and the Chair of the IEEE SMC Intelligent Power and Energy Systems Technical Committee. He is an IET Member and a Chartered Engineer.

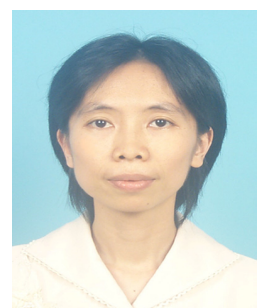

Ping Yang (M'11) received the Ph.D. degree in automatic control from South China University of Technology, Guangzhou, China, in 1998. She is currently a Professor with the School of Electric Power Engineering, South China University of Technology, Guangzhou, China and the Director of Guangdong Key Laboratory of Clean Energy Technology, South China University of Technology, Guangzhou, China. Her current research interests include smart microgrid and electricity market. 


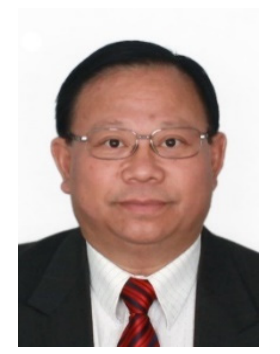

Loi Lei Lai (M'87, SM'92, F'07, LF'21) received the B.Sc. (First Class Hons.), Ph.D., and D.Sc. degrees in electrical and electronic engineering from the University of Aston, Birmingham, UK, and City, University of London, London, UK, in 1980, 1984, and 2005, respectively.

Professor Lai is currently a University Distinguished Professor with Guangdong University of Technology, Guangzhou, China. He was a Pao Yue Kong Chair Professor with Zhejiang University, Hangzhou, China, and the Professor and Chair of Electrical Engineering with City, University of London. His current research areas are in smart cities and smart grid. Professor Lai was awarded an IEEE Third Millennium Medal, the IEEE Power and Energy Society (IEEE/PES) UKRI Power Chapter Outstanding Engineer Award in 2000, the IEEE/PES Energy Development and Power Generation Committee Prize Paper in 2006 and 2009, the IEEE/SMCS Outstanding Contribution Award in 2013 and 2014, the Most Active Technical Committee Award in 2016, and his research team has received a Best Paper Award in the IEEE International Smart Cities Conference in October 2020. Professor Lai is an Associate Editor of the IEEE Transactions on Systems, Man, and Cybernetics: Systems, Editor-in-Chief of the IEEE Smart Cities Newsletter, a member of the IEEE Smart Cities Steering Committee and the Chair of the IEEE Systems, Man, and Cybernetics Society (IEEE/SMCS) Standards Committee. He was a member of the IEEE Smart Grid Steering Committee; the Director of Research and Development Center, State Grid Energy Research Institute, China; a Vice President for Membership and Student Activities with IEEE/SMCS; and a Fellow Committee Evaluator for the IEEE Industrial Electronics Society. He is a Fellow of IET.

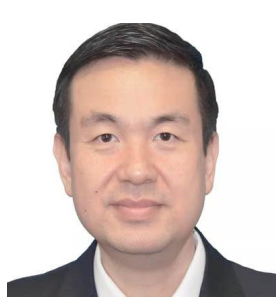

Peng Li received the B.Sc., M.Sc., and Ph.D. degrees from South China University of Technology, Guangzhou, China, in 1993, 1995, and 2002, respectively, and the Ph.D. degree from the Technical University of Braunschweig, Braunschweig, Germany, in 2004, all in electrical engineering. He is currently a Professor-level Senior Engineer of the Digital Grid Research Institute of China Southern Power Grid, Guangzhou, China. His current research interests include technology research and management of smart grid and digital grid.

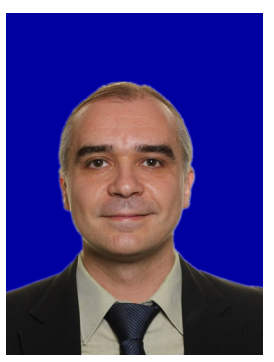

Josep M. Guerrero (S'01-M'04-SM'08FM'15) received the B.Sc. degree in telecommunications engineering, the M.Sc. degree in electronics engineering, and the Ph.D. degree in power electronics from the Technical University of Catalonia, Barcelona, in 1997, 2000 and 2003, respectively. Nowadays he is working towards the M.Sc. Degree in Psychobiology and Cognitive Neuroscience at the Autonomous University of Barcelona.
Since 2011, he has been a Full Professor with AAU Energy, Aalborg University, Denmark, where he is responsible for the Microgrid Research Program. From 2019, he became a Villum Investigator by The Villum Fonden, which supports the Center for Research on Microgrids (CROM) at Aalborg University, being Prof. Guerrero the founder and Director of the same center (www.crom.et.aau.dk).

His research interests is oriented to different microgrid frameworks in applications like microgrid clusters, IoT-based and digital twin, maritime microgrids for electrical ships, vessels, ferries and seaports, and space microgrids applied to nanosatellites and closed ecological systems. Prof. Guerrero is an Associate Editor for a number of IEEE TRANSACTIONS. He has published more than 800 journal papers in the fields of microgrids and renewable energy systems, which are cited more than 70,000 times. During eight consecutive years, from 2014 to 2021, he was awarded by Clarivate Analytics (former Thomson Reuters) as Highly Cited Researcher with 50 highly cited papers. In 2021, he received the IEEE Bimal Bose Award for Industrial Electronics Applications in Energy Systems, for his pioneering contributions to renewable energy based microgrids.

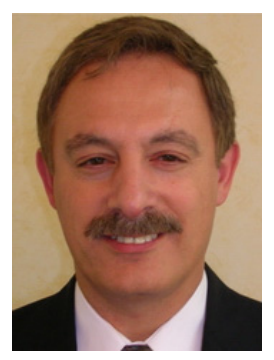

Mohammad Shahidehpour (Life Fellow, IEEE) received the Honorary Doctorate degree from the Polytechnic University of Bucharest, Bucharest, Romania. He is a University Distinguished Professor, the Bodine Chair Professor, and the Director of the Robert W. Galvin Center for Electricity Innovation, Illinois Institute of Technology. He is also a Research Professor with the Center of Research Excellence in Renewable Energy and Power Systems, King Abdulaziz University, Jeddah, Saudi Arabia. He was the recipient of the 2019 IEEE PES Ramakumar Family Renewable Energy Excellence Award. He is a member of the U.S. National Academy of Engineering. He is a Fellow of the American Association for the Advancement of Science and the National Academy of Inventors. 